\title{
Medical Problems during Participation of Medical Congress - A Long Trip to Val D'Isere from Korea
}

\author{
Darlene Park, MD, Minjae Kim, MD, Ga Young Lee, BS, \\ Ku Hyun Yang, MD, Hye Sun Park, MD, Dae Chul Suh, MD
}

Since the 3rd WIN meeting in 1982 more than 500 participants join the meeting in Val D'Isere every year [1]. One of our authors has attended the meeting more than 10 times. He experienced many physical illnesses while travelling from South Korea to Val D'Isere in France, which is located in the Alps mountain near the border between France and Italy. In order to get there, it is necessary to take airplane, train, and/or bus with a heavy suitcase. During the trip which usually takes more than 15 hours, he experienced headache, gastrointestinal trouble, sleep disturbance and other additional physical illnesses. Therefore, we reviewed the itinerary to Val D'Isere and presented physical illnesses which occurred during a long trip for an academic activity by specialized professionals such as university hospital professors. In addition, we discussed the mechanism of such illnesses and offered possible solutions including medical treatment.

\section{Key Words : Travel medicine; Neurointervention; Medical congress}

\footnotetext{
All authors: Department of Radiology, University of Ulsan, College of Medicine, Asan Medical Center, Seoul, Korea

Received February 24, 2016;

accepted after revision February 29, 2016.

Correspondence to: Dae Chul Suh, MD, Department of Radiology, University of Ulsan, College of Medicine, Asan Medical Center, 88, Olympic-ro 43-gil, Songpa-gu, Seoul 138-736, Korea.

Tel. 82.2.3010.4366 Fax. 82.2.476.0090

E-mail: dcsuh@amc.seoul.kr

This is an Open Access article distributed under the terms of the Creative Commons Attribution Non-Commercial License (http://creativecommons.org/licenses/by-nc/3.0) which permits unrestricted non-commercial use, distribution, and reproduction in any medium, provided the original work is properly cited.
}

There are traditional risks related to international trips for academic activities which may result in a heart condition or reduce traveller's own performance. A wide variety of health problems including gastrointestinal (GI) trouble such as abdominal pain, diarrhea, headache, sleep disturbance, and motion sickness have been reported [2]. Most of these are related to many factors such as travel duration, quality of accommodation and transportation, oral intake and traveler's own health status. However, we can overcome and improve the experience by taking precursory measures to prevent and control some, if not all, of these conditions.

The purpose of this article is to describe the spectrum of travel-related illnesses that can happen during international academic trips and to raise awareness of possible measures and offer tips to prevent and control these illnesses to maintain a good physical and mental well-being and improve performance during academic activities. Immunization for infectious disease or prevention of any condition related with infectious diseases may be required but this is beyond the subject of this study because the congress of concern in this study is usually held in areas where such vaccination is not necessary.

\section{Travel course from Seoul to Val D'Isere}

Val D'Isere is located in the Rhone-Alpes region (Savoie department) in the southeastern France near to the border of Italy (Fig. 1). Val D'Isere is known as a vacation spot especially in the winter as well as the host of the winter Olympics in 1992. There are two ways from Seoul to get to Val D'Isere: one by bus through Geneva and one by train via Paris (Fig. 1). Several 
reservation information was listed in a blog [3]. Because there is currently no direct flight from Seoul to Geneva, it is necessary to stopover in Paris or an alternative city. Flight time to Paris is usually about 12 hours and it takes about 1 hour to fly from Paris to Geneva. It takes 4 hours from Geneva international airport to Val 'Isere by bus.

There are two ways of getting a train from Paris to Val D'Isere; one by daytime train and the other by night train. If taking a route by any trains, additional 30 minute bus drive is necessary to travel from BourgSaint Maurice to Val I'Isere. Therefore, an overnight stay in Paris needs to be considered in order to get to Val Isere on time or in an appropriate time to get the bus in Bourg-Saint Maurice or to check in hotel in Val I'Disere.

\section{Common medical problems related with a long trip to Val D'Isere}

Medical illnesses related to the long trip to Val D'Isere can occur during travelling via flight, train or bus and during the meeting. Respiratory difficulty, nausea or general weakness can occur in high altitude as in the Alps. Frostbite or accident can be other problems because it is generally very cold with frequent heavy snow in Val D'Isere that is located in high altitude. The most common problems in an airplane were syncope or presyncope (37.4\% of cases), respiratory symptoms $(12.1 \%)$, and nausea or vomiting $(9.5 \%)$ [4]. Another report showed that the most common medical events were transient loss of consciousness (41\%), nausea/vomiting/diarrhea (19.5\%), and breathing difficulty (16\%) [5]. Those events were potentially associated with the stresses of flight; for example, loss of consciousness or syncope may be associated with mild hypoxia and immobility; nausea and vomiting and shortness of breath were likely to be associated with a degree of anxiety-related hyperventilation [5].

There are some interesting findings that in frequent international business travelers short trips cause more problems. More specifically, having higher alcohol consumption over the recommended limit and failing to get the recommended amount of sleep ( 8 hours per night for an adult) will aggravate the risk of developing such symptoms [6]. Younger travellers fell ill to a greater extent than older travellers. The fact that younger travellers are at an increased risk of travelrelated illnesses confirms the results of previous studies [7]. The main illnesses described here included gastrointestinal trouble, headache and sleep disturbance because one of the authors (Suh DC) has experienced similar patterns almost every year.

\section{GI trouble}

GI trouble can occur during flight or at the destination and includes various symptoms including abdominal pain, diarrhea, nausea or vomiting. Heavy meal or excessive alcohol intake, ingestion of cold food or unfamiliar food can be the cause of the GI trouble. The
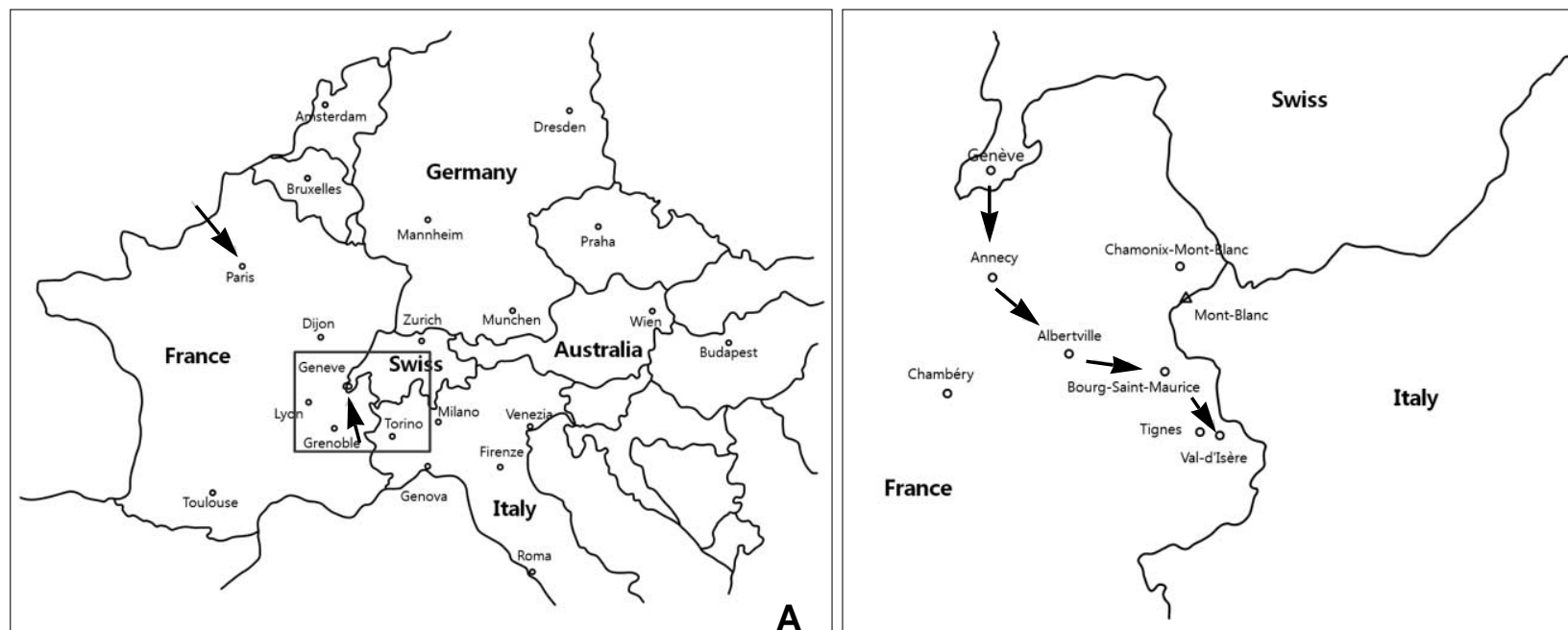

Fig. 1. Map of European countries (A) to show how to get to the French Alps. Rectangular box is magnified in (B). After flight to Paris (a long arrow in $\mathbf{A}$ ), there is a train (day or night trains) at the Gare D'Austeriz to get to Bourg-Saint-Maurice. After flight to Genève (a short arrow in $\mathbf{A}$ ), there is a bus to get to Val D'lsere. Close-up (B) of the Savoi region (rectangular box in Fig. 1A) shows where Val D'lsere located. Arrows indicate travel course from Genève to Val D'lsere by bus. It takes about 4 hours. 
GI system expands by $25 \%$ under the lower atmospheric pressure in the flight and the oxygen level is lower-[8]. Passengers with constipation can suffer from abdominal pain or distention during the flight. For prevention, it is important to wear comfortable clothes and refrain from excess consumption of food and soft drink. Most people who develop an episode of traveler's diarrhea experience a self-limiting illness with approximately 1 day of disability without further medical complications [9].

Alcohol consumption was more likely on longer flights, when traveling with friends, and anticipating First or Business Class seating [10]. Passengers in an airplane became intoxicated approximately 3 times faster. In the case of drinking gas-evaporating beer or champaign, abdominal distension was frequently reported. This is particularly important for the elderly and patients with chronic disease such as diabetes, and coronary heart disease. Cold food and beverage including dairy products sometimes evoke discomfort of the bowels and also may cause diarrhea. In order to prevent GI trouble, efforts not to consume excess food and alcohol and to avoid cold food and beverage would be helpful [11].

\section{Headache}

According to an author's experience, headache appeared in the airplane or at the destination after alcohol drinking. The most common and serious situation was headache after drinking of several different kinds of liquor including wines. Different kinds of wine, champaign or beer might aggravate the headache particularly at a destination with high altitude which promotes rapid absorption of alcohol. Sweet things such as chocolate and dairy products such as cheese seemed to be related to headache if taken in a large amount.

Airplane headache is usually a symptom caused by the exposure to rapid change and variation of atmospheric pressure rather than high altitude [12]. The characteristics of pain are diffuse severe, fronto-orbital pain, lasting less than 20 minutes, typically associated with take-off or landing. It is known to be due to failing of the compensation between intra-sinus and external pressure causing barotrauma, which affects ethmoidal nerves from the ophthalmic branch of the trigeminal nerve [13]. Preventative measures are nonpharmacological pressure on the painful area, Valsalva maneuver, increasing fluid intake, reducing amount of alcohol, or taking nonsteroidal anti-inflammatory drugs as pharmacological treatment [14],

The flight motion sickness may be the most common symptom when people first get on the flight with feeling of dizziness, nausea, and sometimes severe vomiting. Relieving the psychological stress seems to be important by meditating, taking a deep breath slowly, and achieving peaceful state of mind. Moreover, fastening the seatbelt and wearing comfortable clothes may be helpful. It happens more severely in the status of overeating, overdrinking and starving [14]. Therefore, it is important to keep a record of oral intake before or on board.

\section{Sleep disturbance}

Comparison of international business travelers with general population revealed that frequent international business travelers were associated with a lower body mass index, lower blood pressure, excess alcohol consumption, sleep deprivation, and diminished confidence to keep up with the pace of work [15]. Circadian or diurnal rhythm is a biological process that displays an endogenous oscillation of about 24 hours. During a trip abroad, there will be a time difference and the circadian rhythm is temporally interrupted. This will inevitably affect sleep pattern or mood state, resulting in sleep disturbance [16]. To overcome this, traveler can adjust exposure time to sunlight to maintain circadian rhythm because the amount of melatonin secretion in the body is controlled by exposure time to the sunlight. Sleeping pill may be helpful but is generally not recommended [15].

A sound sleep is required especially when an important presentation is scheduled the next day. In those situations, minimal amount of sleeping pill would be enough to adapt to jet lag if there is a sleep disturbance. One of the authors could sleep well after taking only a small dose of sleeping pill (a half of Zolpidem $6 \mathrm{mg}$ tablet). One of the authors experienced that putting a bandage over his eyes induced a good quality sleep in the airplane or at night because various light sources hinder a good quality sleep.

\section{Homesickness}

Homesickness defined as the distress or impairment caused by an actual or anticipated separation from home with preoccupying thoughts of home and attachment objects. Homesickness can make travelers experience depression and anxiety, withdrawn behavior, and difficulty focusing on topics unrelated to home. 
However, homesickness about food of his or her hometown and social customs are particularly problematic when travelers cannot adjust the local food. Strategies to overcome this include combinations of environmental information, psycho-education, social support, explicit coping instruction, caregiver education, practice time away from home, and surrogate caregiver training.

\section{Conclusions}

GI trouble, headache and sleep disturbance were the most common travel-related illnesses associated with a long trip such as from South Korea to Val D'Isere for an academic congress. Although good meals and beverages are required for a pleasant journey excessive consumption of food and alcohol can disturb the physical and mental wellbeing of travelers. Therefore, refraining and exerting self-control regarding food and alcohol and avoiding cold and unfamiliar food are needed. Sleep disturbance due to jet lag can be an obstacle in preparing for and performing academic activities such as presentation and discussion at the congress. Although various preventative and conservative measures can be employed to maintain both physical and mental wellbeing, some medication may be required if not managed conservatively.

\section{References}

1. Picard L. The history of ABC WIN international neuroradiology in Val D'Isere 35th birthday. 2016;13

2. Hill DR, Pearson RD. Health advice for international travel. Ann
Intern Med 1988;108:839-852

3. http://blog.naver.com/neurostyle/50154188491 accessed on Feb. 13,2016

4. Peterson DC, Martin-Gill C, Guyette FX, Tobias AZ, McCarthy CE, Harrington ST, et al. Outcomes of medical emergencies on commercial airline flights. N Engl J Med 2013;368:2075-2083

5. Mahony PH, Myers JA, Larsen PD, Powell DM, Griffiths RF. Symptom-based categorization of in-flight passenger medical incidents. Aviat Space Environ Med 2011;82:1131-1137

6. Al-Zurba F, Saab B, Musharrafieh U. Medical problems encountered among travelers in Bahrain International Airport clinic. $J$ Travel Med 2007;14:37-41

7. Angelin M, Evengard B, Palmgren H. Travel health advice: benefits, compliance, and outcome. Scand J Infect Dis 2014;46:447-453

8. Connor BA. Chronic diarrhea in travelers. Curr Infect Dis Rep 2013;15:203-210

9. Dupont HL. Chronic complications after travelers' diarrhea. $J$ Travel Med 2013;20:273-274

10. Girasek DC, Olsen CH. Airline passengers' alcohol use and its safety implications. J Travel Med 2009;16:311-316

11. Nair D. Travelers' diarrhea: prevention, treatment, and post-trip evaluation. J Fam Pract 2013;62:356-361

12. Mainardi F, Lisotto C, Maggioni F, Zanchin G. Headache attributed to airplane travel ('airplane headache'): clinical profile based on a large case series. Cephalalgia 2012;32:592-599

13. Mainardi F, Zanchin G. "Airplane headache” or flight-related headache? Cephalalgia 2011;31:254-255

14. Mainardi F, Maggioni F, Lisotto C, Zanchin G. Diagnosis and management of headache attributed to airplane travel. Curr Neurol Neurosci Rep 2013;13:335

15. Burkholder JD, Joines R, Cunningham-Hill M, Xu B. Health and well-being factors associated with international business travel. $J$ Travel Med 2010;17:329-333

16. Weingarten JA, Collop NA. Air travel: effects of sleep deprivation and jet lag. Chest 2013;144:1394-1401 